\title{
Polaron spin dynamics in high-mobility polymeric semiconductors
}

Sam Schott, Uday Chopra, Vincent Lemaur, Anton Melnyk, Yoan Olivier, Riccardo DiPietro, Igor Romanov, Remington Carey, Xuechen Jiao, Cameron Jellett, Mark Little, Adam Marks, Christopher R. McNeill, Iain McCulloch, Erik R. McNellis, Denis Andrienko, David Beljonne, Jairo Sinova, Henning Sirringhaus*

*hs220@cam.ac.uk

\section{Contents}

1 Sample manufacturing $\quad 1$

2 Gate-voltage-dependent mobilities $\quad 3$

3 Spin counting $\quad 4$

4 Determination of spin lifetimes $\quad \mathbf{5}$

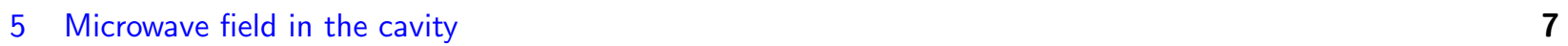

6 The Einstein relation for charge and spin transport $\quad 8$

7 Hall effect measurements in IDTBT $\quad 9$

8 DFT calculation of spin admixtures $\quad 11$

9 Estimate of intra-site relaxation in IDTBT 12

10 GIWAXS measurements $\quad 13$

11 Raman measurements $\quad 14$

12 Torsional autocorrelation functions $\quad 15$

13 Dynamical disorder in IDTBT 16

\section{Sample manufacturing}

The geometry of the ESR cavity results in a maximum of the microwave (mw) magnetic field $B_{\mathrm{mw}}$ in a narrow volume around the cavity center and requires a matching device design. At the same time, we aim to maximize the FET active area in order to increase the number of spins and thus the ESR signal. Our transistor design with an active area of $3 \mathrm{~mm}$ width and $20 \mathrm{~mm}$ height therefore limits the device to a volume where $B_{\mathrm{mw}}$ is dominant over the electric component while maintaining a large total area. Such an asymmetric layout naturally poses problems for spin-coating, the method of choice for polymer thin film deposition. It is thus preferable to manufacture multiple FETs on one large substrate and to separate them after processing.

We use $0.5 \mathrm{~mm}$ thick and $40 \mathrm{~mm} \times 40 \mathrm{~mm}$ wide Suprasil quartz slides as bottom substrates. To facilitate the separation of FETs after processing, we pre-cut $0.3 \mathrm{~mm}$ deep grooves into the substrate back with a diamond saw. A custom-designed vacuum chuck with a hole pattern matching the grooves is used to hold the substrate during spin-coating. 
quartz glass with grooves

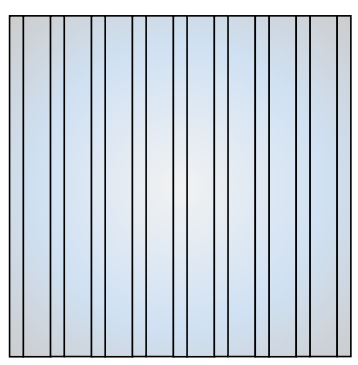

8 FETs per $40 \times 40 \mathrm{~mm}^{2}$ quartz substrate

lithography and spin-coating process, gate evaporation
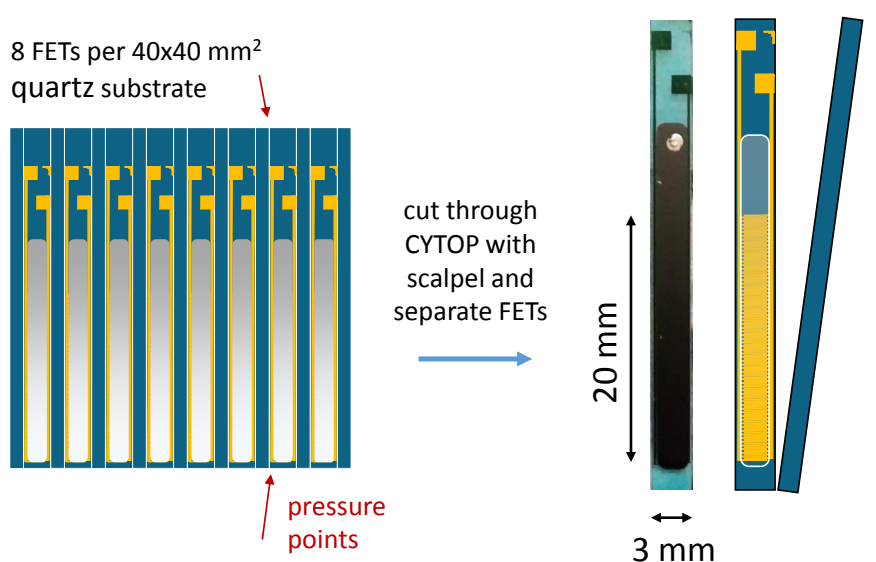

Figure S1: Manufacturing process of IDTBT transistors for FI-ESR on top of $40 \mathrm{~mm} \times 40 \mathrm{~mm}$ quartz slides. The photograph on the right hand shows a real device.

On top of such prepared substrates, we proceed with a standard top-gate bottom-contact FET manufacturing process. We use interdigitated source and drain electrodes made out of gold with the design shown in Fig. S1. The channel length and width are $L=100 \mu \mathrm{m}$ and $W=243 \mathrm{~mm}$, respectively, with an active area size of $2.45 \mathrm{~mm} \times 20 \mathrm{~mm}$. Contact pads for electrical connections are located at the top of the sample, about $10 \mathrm{~mm}$ away from the active area, to be placed outside the mw cavity.

Chromium / gold $(4 \mathrm{~nm} / 20 \mathrm{~nm})$ electrodes are lithographically patterned onto the quartz with a double-layer lift-off process. The active polymer layer and dielectric are successively spin-coated on top and annealed with deposition parameters depending on the materials choice. Finally, a $25 \mathrm{~nm}$ thick aluminum electrode is deposited through shadow-mask-evaporation as a top gate. The finished devices are then separated by applying a small amount of manual pressure to the pre-cut grooves.

The devices are mounted on top of a quartz glass carrier with copper tracks for the electrode connections as shown in Fig. S2 and contacted though wire bonding. This results in electrical connections which remain stable down to $5 \mathrm{~K}$ and over multiple temperature cycles while keeping the bulk of the electrical connections (soldering points, copper tracks) outside of the cavity. The device electrodes themselves are much thinner than the skin depth of microwaves of ca. $800 \mathrm{~nm}$ at $9 \mathrm{GHz}$ and therefore have minimal impact on the resonance properties of the cavity.

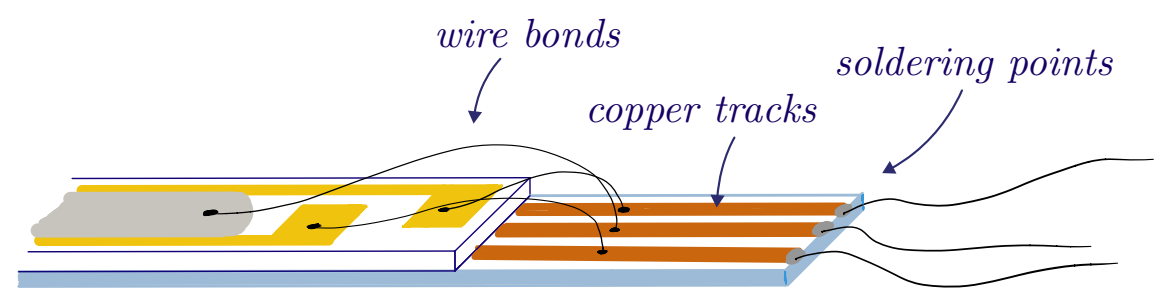

Figure S2: Schematic of electrical contacts to a FI-ESR device. 


\section{Gate-voltage-dependent mobilities}

Charge carrier mobilities can be determined from FET transfer characteristics in the linear and saturation regimes. The current flowing though an FET channel under application of a drain voltage $V_{\mathrm{D}} \leq V_{\mathrm{G}}-V_{\mathrm{th}}$ depends on the mobility $\mu$ through $^{1,2}$

$$
I_{\mathrm{SD}}=\frac{W}{L} \mu C_{\mathrm{i}}\left(\left(V_{\mathrm{G}}-V_{\mathrm{th}}\right) V_{\mathrm{D}}-\frac{1}{2} V_{\mathrm{D}}^{2}\right)
$$

Here, $C_{i}$ is the dielectric capacitance per unit area and $W$ and $L$ are the channel width and length, respectively. $V_{\mathrm{th}}$ is the threshold voltage that needs to be overcome for the FET to switch "on".

When increasing the drain voltage beyond $V_{\mathrm{G}}-V_{\mathrm{th}}$, the carrier concentration is pinched off at the drain electrode and the FET is said to operate in the saturation regime. Eq. (S1) remains valid by substituting $V_{\mathrm{D}}=V_{\mathrm{G}}-V_{\mathrm{th}}$ :

$$
I_{\mathrm{SD}}^{\mathrm{sat}}=\frac{W}{2 L} \mu C_{\mathrm{i}}\left(V_{\mathrm{G}}-V_{\mathrm{th}}\right)^{2} .
$$

Equations (S1) and (S2) can be used to extract the charge carrier mobilities in the linear and saturation regime, respectively. Simple differentiation gives

$$
\begin{aligned}
\mu_{\text {lin }} & =\frac{L}{W C_{\mathrm{i}} V_{\mathrm{D}}} \frac{\partial I_{\mathrm{SD}}}{\partial V_{\mathrm{G}}} & \text { for } & V_{\mathrm{D}} \leq V_{\mathrm{G}}-V_{\mathrm{th}} \\
\mu_{\mathrm{sat}} & =\frac{2 L}{W C_{\mathrm{i}}}\left(\frac{\partial \sqrt{I_{\mathrm{SD}}}}{\partial V_{\mathrm{G}}}\right)^{2} & \text { for } & V_{\mathrm{D}} \geq V_{\mathrm{G}}-V_{\mathrm{th}} .
\end{aligned}
$$

We typically record two transfer curves to calculate mobilities, one at $V_{\mathrm{D}}= \pm 5 \mathrm{~V}$ and one at $V_{\mathrm{D}}= \pm 60 \mathrm{~V}$, and sweep the gate voltage up to $V_{\mathrm{G}}= \pm 60 \mathrm{~V}$. When extracting the mobility from the first curve in the interval from $\pm 40 \mathrm{~V}$ to $\pm 60 \mathrm{~V}$, we are well within the linear regime. For the second curve, we are in saturation only.

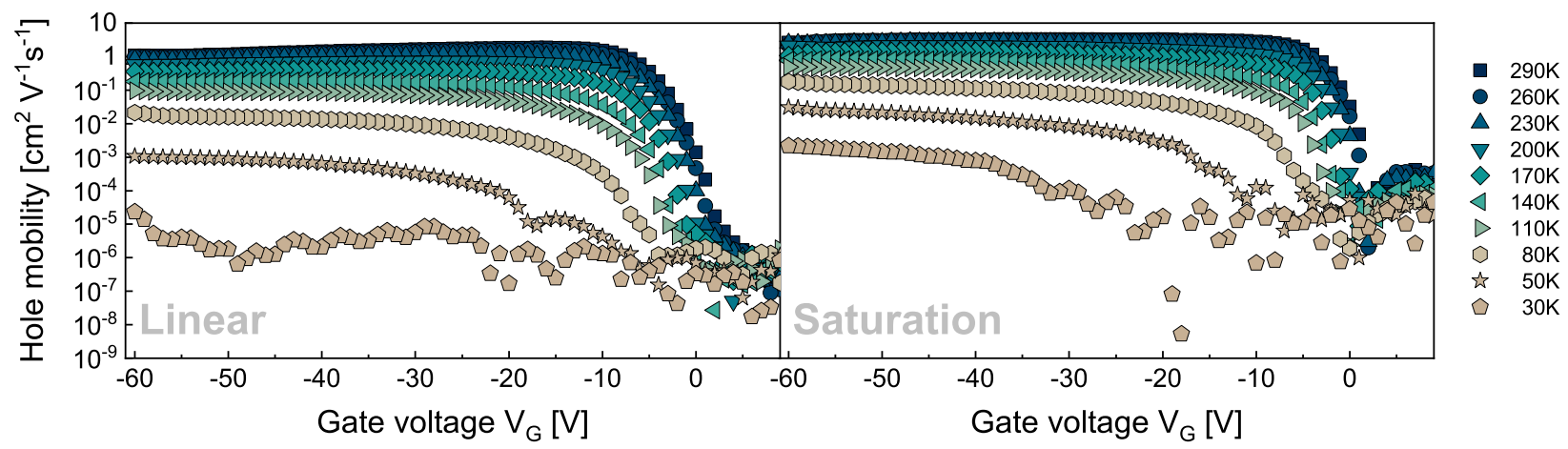

Figure S3: Hole mobilities in the linear and saturation regimes $\mu_{\text {lin }}$ and $\mu_{\text {sat }}$ with $V_{\mathrm{D}}=-5 \mathrm{~V}$ and $-60 \mathrm{~V}$, respectively, from $290 \mathrm{~K}$ to $30 \mathrm{~K}$. The mobility becomes gate-voltage-independent from 170-200 K. 


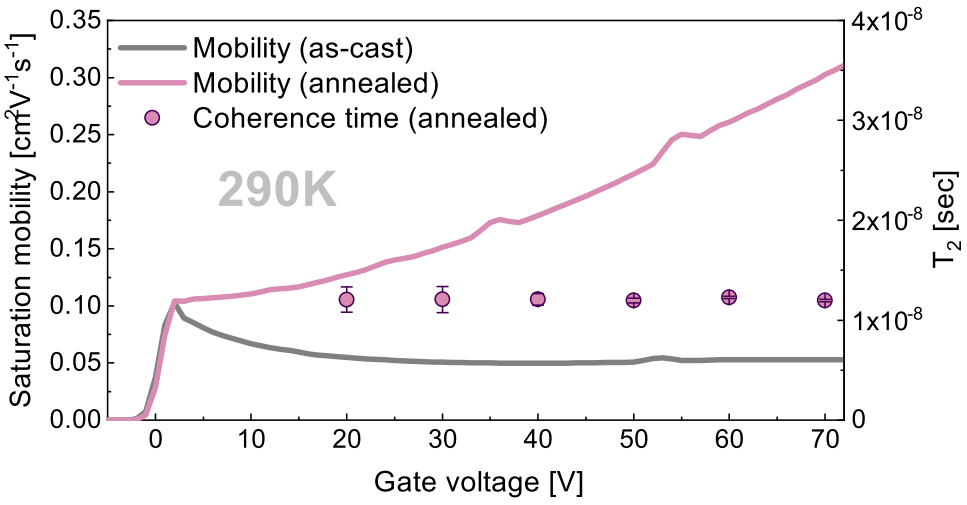

Figure S4: Gate-voltage-dependence of the electron mobility in FI-ESR devices with as-cast and annealed $\mathrm{p}$ (NDI2O-T2) at room temperature (left axis) and gate-voltage-dependence of the coherence time for annealed $\mathrm{p}$ (NDI2O-T2) (right axis).

\section{Spin counting}

The magnetic susceptibility of a sample can be determined from its integrated ESR absorption spectrum or double-integrated 1st harmonic spectrum $\mathrm{as}^{3}$

$$
\chi=k \cdot \mathrm{DI} \frac{\mu_{0} \hbar^{2} \gamma_{\mathrm{e}}^{2}}{3 \hbar \omega_{\mathrm{mw}} Q \sqrt{P_{\mathrm{mw}}} B_{\mathrm{mod}} \overline{f(y)}}
$$

where DI is the double integrated intensity, normalized for integration time and receiver gain, $\omega_{\text {res }}=2 \pi f_{\text {res }}$ is the mw frequency, $Q$ denotes the cavity quality factor (Q-value), and $P_{\mathrm{mw}}$ is the mw power. $k$ is a calibration factor specific to the spectrometer properties and detection electronics and $\overline{f(y)}$ is a correction factor accounting for the vertical sample position in the cavity. Therefore, $\overline{f(y)}=1$ for a point sample at the center of the cavity and $\overline{f(y)}=0$ for a sample outside of the cavity. The calculation of $\overline{f(y)}$ is discussed in Supplementary Note 5 . The Q-value is given by the ratio of the cavity's resonance frequency divided by the full-width-at-half-maximum (FWHM) of its frequency dependent absorption profile

$$
Q=\frac{\omega_{\mathrm{res}}}{\omega_{\mathrm{FWHM}}}
$$

and is determined from a least-squares fit of the latter.

With a known magnetic susceptibility, the number of spins can be determined from an appropriate model for the magnetization of the sample in an external field. For charge carrier spins in a semiconductor with a Fermi level close to the band edge $\left(E_{\mathrm{F}}-E_{\mathrm{B}} \ll k_{\mathrm{B}} T\right)$, one expects a Curie susceptibility

$$
\chi_{0}=\mu_{0} \frac{\hbar^{2} \gamma_{\mathrm{e}}^{2}}{3 k_{\mathrm{B}} T} S(S+1) n_{\mathrm{spin}}
$$

where $S=1 / 2, \gamma_{\mathrm{e}}^{2}$ denotes the gyromagnetic ratio of an electron, $2 \pi \hbar$ the Planck constant, $\mu_{0}$ the vacuum permeability, $k_{\mathrm{B}}$ the Boltzmann constant, and $T$ the temperature. 


\section{Determination of spin lifetimes}

Charges in organic semiconductors are, over a wide temperature range, sufficiently mobile for motional narrowing to average over local hyperfine and spin-orbit fields. Under such conditions, the ESR absorption signal is given by a single Lorentzian ${ }^{4}$

$$
\begin{aligned}
L\left(B, B_{\mathrm{mw}}\right) & \propto \chi_{0} \frac{\omega B_{\mathrm{mw}} T_{2}}{1+\gamma_{\mathrm{e}}^{2} T_{2}^{2}\left(B-B_{\mathrm{res}}\right)^{2}+\gamma_{\mathrm{e}}^{2} T_{1} T_{2} B_{\mathrm{mw}}^{2}} \\
& \simeq \chi_{0} \frac{\omega B_{\mathrm{mw}} T_{2}}{1+\gamma_{\mathrm{e}}^{2} T_{2}^{2}\left(B-B_{\mathrm{res}}\right)^{2}}
\end{aligned}
$$

where Eq. (S9) holds for $\gamma_{\mathrm{e}}^{2} T_{1} T_{2} B_{\mathrm{mw}}^{2} \ll 1$. The coherence time $T_{2}$ can therefore be extracted directly from the ESR line width if the system is not in saturation while the spin-lattice relaxation time $T_{1}$ can be determined from the saturation behavior under increasing microwave power.

In continuous-wave ESR, signals are detected with a lock-in amplifier where the external magnetic field is modulated at a frequency $\omega_{\text {mod }}$ and a peak-to-peak amplitude $B_{\text {mod }}$ :

$$
B(t)=B_{0}+\frac{B_{\mathrm{mod}}}{2} \sin \left(\omega_{\bmod } t\right) .
$$

The signal is then detected as a first or second harmonic of $\omega_{\text {mod }}$. The recorded signal will be proportional to the change in mw absorption during a modulation cycle. Choosing a larger modulation amplitude $B_{\text {mod }}$ will therefore result in a stronger signal. If the modulation amplitude approaches the signal linewidth, however, the ESR lineshape can no longer be approximated as the derivative of the absorption signal and distortion due to field modulation needs to be explicitly taken into account. To improve the signal-to-noise ratio, we were often forced to choose values of $B_{\text {mod }}$ that approach the smallest features in the spectrum and account for the resulting distortions in line shape during fitting.

The effects of field modulation and $n$-th harmonic detection can be modeled as a convolution of $S\left(B_{0}\right)$ with a modulation kernel $^{5,6}$

$$
M_{n}\left(B_{0}, B_{\text {mod }}\right)=i^{n} \int_{-\infty}^{\infty} J_{n}\left(B_{\bmod } \beta / 2\right) \mathrm{e}^{i B_{0} \beta} \mathrm{d} \beta
$$

where $J_{n}(x)$ is a $n$-th order Bessel function of the first kind. The the $n$-th harmonic signal (with the time dependence dropped) is then given by

$$
s_{n}\left(B_{0}, B_{\mathrm{mod}}\right)=M_{n}\left(B_{0}, B_{\mathrm{mod}}\right) * S\left(B_{0}\right) .
$$

The above equation is implemented in MATLAB to reproduce the experimental spectra: the expected curve for specific lifetimes is Fourier transformed, multiplied with $J_{1}\left(B_{\bmod } \beta / 2\right)$ and transformed back before fitting to the spectrum. Since the modulation amplitude is known from the selected acquisition parameters, the resulting signal distortions are fully taken into account.

Determining both $T_{1}$ and $T_{2}$ requires the knowledge of the saturation behavior of the spectrum under sufficiently high mw powers. This results from the fact that $S\left(B_{0}\right)$ is independent of $T_{1}$ in the absence of saturation. The common approach in literature has been to calculate the integrated 
area of spectra for different mw powers individually and then fit the power dependence of this area to the expected saturation behavior:

$$
A\left(B_{\mathrm{mw}}\right) \propto \chi_{0} \frac{\pi}{\gamma_{\mathrm{e}}} \frac{B_{\mathrm{mw}}}{\sqrt{1+\gamma_{\mathrm{e}}^{2} T_{1} T_{2} B_{\mathrm{mw}}^{2}}} .
$$

This method, applied to typically noisy FI-ESR spectra, has the downside of large fitting errors for spectra at either very small or large mw powers. This is shown for a simulated power saturation measurement in Fig. S5a with artificially introduced $1 / f$ noise to give a signal-to-noise $(\mathrm{S} / \mathrm{N})$ ratio of $5 \mathrm{~dB}$. Calculating the integrated areas separately for every slice from a least-squares Lorentzian fit and extracting the lifetimes from Eq. (S13) yields a $T_{1}$ value which is under-estimated by $80 \%$ and a deviation in $T_{2}$ of $10 \%$ (see Fig. S5b).
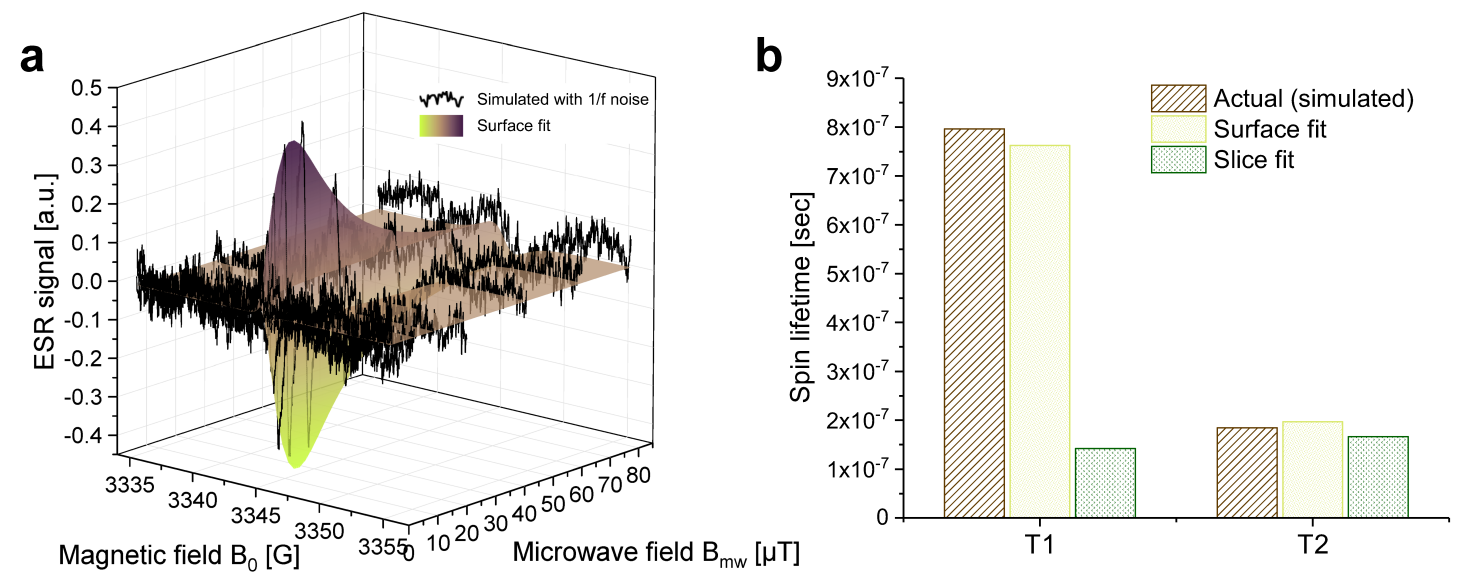

Figure S5: Fitting a power saturation measurement in the presence of noise. (a) Simulated ESR spectra for a Lorentzian resonance with lifetimes of $T_{1}=0.8 \mu \mathrm{s}$ and $T_{2}=0.2 \mu \mathrm{s}$ for increasing mw fields. $1 / f$ noise with a $\mathrm{S} / \mathrm{N}$ ratio of $5 \mathrm{~dB}$ has been artificially added and the resulting spectra are fitted as a single 2D data set to Eq. (S14). (b) Comparison of actual lifetimes used for the simulation and lifetimes extracted from the $2 \mathrm{D}$ surface fit and the individual slice fits, respectively.

We propose instead to fit the full power saturation measurement, i.e., all spectra at once, to the expected saturation behavior from Eq. (S8):

$$
\begin{aligned}
s\left(B_{0}, B_{\mathrm{mw}}\right) & =M_{1}\left(B_{0}, B_{\mathrm{mod}}\right) * L\left(B_{0}, B_{\mathrm{mw}}\right) \\
& \propto M_{1}\left(B_{0}, B_{\mathrm{mod}}\right) * \chi_{0} \frac{\omega B_{\mathrm{mw}} T_{2}}{1+\gamma_{\mathrm{e}}^{2} T_{2}^{2}\left(B_{0}-B_{\mathrm{res}}\right)^{2}+\gamma_{\mathrm{e}}^{2} T_{1} T_{2} B_{\mathrm{mw}}^{2}} .
\end{aligned}
$$

Such a 2D surface fit uses the stronger signal from intermediate mw powers to stabilize the fit of noisy spectra at very small and large powers. Such a fitted surface is shown in Fig. S5a and the fitted $T_{1}$ and $T_{2}$ values deviate by only $4 \%$ and $7 \%$, respectively, from the actual values used to create the data set. Especially the extracted $T_{1}$ value is significantly more accurate despite the high noise level.

A single Lorentzian fit is no longer possible when the resonance line is inhomogeneously broadened, for example at sufficiently low temperatures where motional narrowing becomes ineffective. In the simplest case, an unresolved splitting from local HFI and SOC fields can be modeled as a 
Gaussian distribution around the center resonance $B_{\text {res }}$ with a standard deviation of $B_{\text {rms }}$ :

$$
G\left(B-B_{\mathrm{res}}\right)=\frac{1}{\sqrt{2 \pi B_{\mathrm{rms}}^{2}}} \exp \left(-\frac{\left(B-B_{\mathrm{res}}\right)^{2}}{2 B_{\mathrm{rms}}^{2}}\right) .
$$

In many cases, all spin packets approximately have the same lifetimes $T_{1,2}$. The absorption signal becomes a convolution of a Gaussian envelope and a Lorentzian line shape, which is a so-called Voigt function:

$$
V\left(B_{0}, B_{\mathrm{mw}}\right)=\int_{-\infty}^{\infty} L\left(B^{\prime}-B_{0}, B_{\mathrm{mw}}\right) G\left(B^{\prime}-B_{\mathrm{res}}\right) \mathrm{d} B^{\prime} .
$$

Qualitatively, the effect of the Gaussian envelop will be to delay the broadening of the resonance with mw power while the integrated area of still saturates with Eq. (S13). In the limit of weak spectral diffusion, the overall power saturation behavior follows the fit function ${ }^{7,8}$

$$
s\left(B_{0}, B_{\mathrm{mw}}\right)=M_{1}\left(B_{0}, B_{\mathrm{mod}}\right) * V\left(B_{0}, B_{\mathrm{mw}}\right)
$$

with five parameters fitting parameters: the spin lifetimes $T_{1,2}$, the inhomogeneous field distribution $B_{\text {rms }}$, the resonance center $B_{\text {res, }}$, and the overall magnitude of the signal, which is proportional to $\chi_{0}$. Only the first three determine the line shape and its saturation behavior, with mutually independent effects, resulting in a very robust fit.

If the envelope function of the spin packet distribution is not known or cannot be easily modeled, it will become necessary again to determine the product of spin lifetimes $T_{1} T_{2}$ from a fit to the integrated area of the spectra. In this case it is essential to achieve a sufficiently high $\mathrm{S} / \mathrm{N}$ ratio to minimize the potentially large errors.

\section{Microwave field in the cavity}

Determining the absolute number of spins and the spin-lattice relaxation time of a sample relies on the knowledge of $B_{\mathrm{mw}}$ over the sample volume while only the applied power $P_{\mathrm{mw}}$ is recorded during an experiment. The scaling of $B_{\mathrm{mw}}$ with $\sqrt{P_{\mathrm{mw}}}$ depends on the resonator architecture and losses inside the cavity.

In the absence of power saturation, the integrated absorption signal of a paramagnetic sample scales only with its magnetic susceptibility and the field amplitude $B_{\mathrm{mw}}$ at the sample position. This allows us to determine $B_{\mathrm{mw}}$ from the integrated absorption signal of a reference sample with a known susceptibility. For the center of the cavity, we find as a function of the cavity Q-value and the incident mw power:

$$
B_{\mathrm{mw}}^{\text {center }}=\eta \cdot \sqrt{Q P_{\mathrm{mw}}}, \eta=2.4069 \times 10^{-6} \mathrm{~T} \mathrm{~W}^{-1 / 2} .
$$

The vertical mw magnetic field distribution $f(y)$ is given by Bruker as a 9th order polynomial and stored with each data acquisition file (Fig. S6a). We additionally confirmed the values by monitoring the ESR signal intensity for a point-like marker sample for different positions in the cavity. Since our samples are confined in a tube with a diameter of $3 \mathrm{~mm}$ around the cavity center, we only correct for the vertical field distribution by calculating the average mw field across the sample length $L$ and neglect any radial variations:

$$
\overline{B_{\mathrm{mw}}(y)}=\frac{1}{L} \int_{-L / 2}^{L / 2} f(y) B_{\mathrm{mw}}^{\text {center }} \mathrm{d} y .
$$



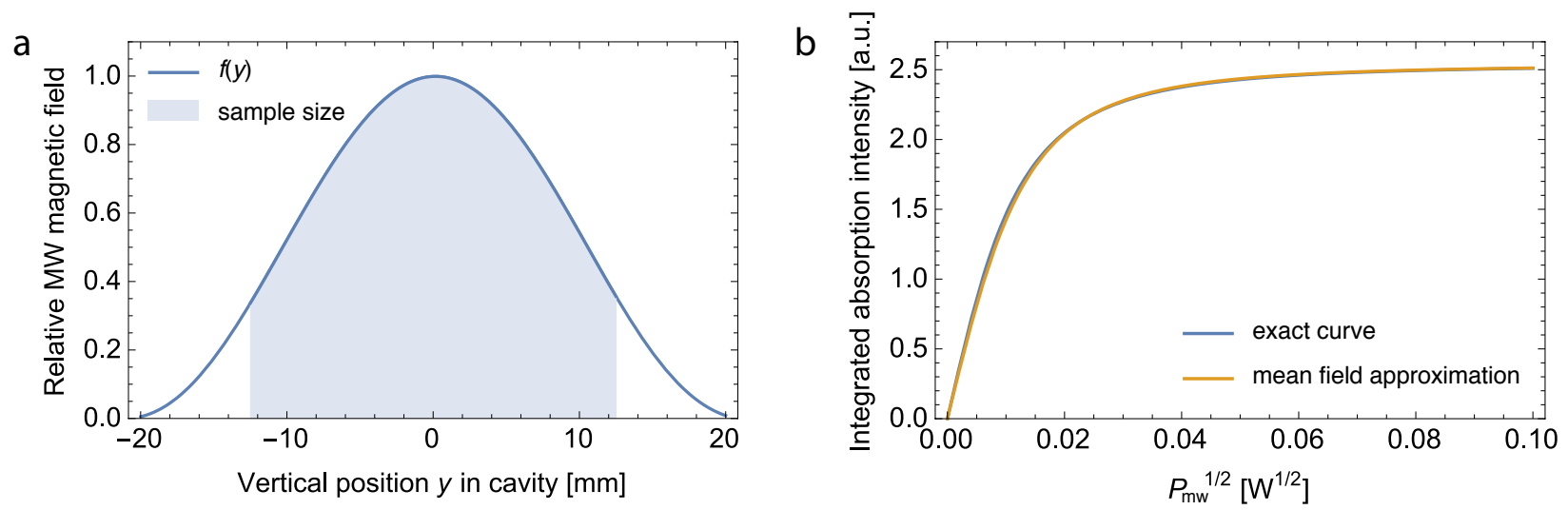

Figure S6: (a) Microwave magnetic field distribution $f(y)$ inside the Bruker ER 4122SHQE cavity. The shaded area is representative of a $25 \mathrm{~mm}$ long sample. (b) Exact power saturation curve from Eq. (S20) taking into account the variation in signal intensity for different positions in the cavity and power saturation behavior of a sample that only sees a uniform average mw field $\overline{B_{\mathrm{mw}}(y)}$. Shown curves are for $L=25 \mathrm{~mm}$ since any visible difference disappears for smaller lengths.

A small error is introduced when using the average field over the sample volume to fit the power saturation behavior because we do not take into account that parts of the sample at positions with a lower field will not only saturate later but also contribute less to the total ESR signal. In fact, the precise expression for the integrated area DI of a spectrum would be

$$
\mathrm{DI}\left(P_{\mathrm{mw}}\right)=\frac{1}{V} \int_{-L / 2}^{L / 2} A\left(f(y) B_{\mathrm{mw}}^{\text {center }}\right) \cdot f(y) \mathrm{d} y \text { with } V=\int_{-L / 2}^{L / 2} f(y) \mathrm{d} y,
$$

where $A$ denotes the integrated absorption spectrum for a single mw field. Our approximation on the other hand gives

$$
\mathrm{DI}\left(P_{\mathrm{mw}}\right) \approx A\left(\overline{B_{\mathrm{mw}}(y)}\right)=\eta A\left(\overline{f(y)} \sqrt{Q P_{\mathrm{mw}}}\right) .
$$

However, the resulting error for our sample length of $L=20 \mathrm{~mm}$ remains well below $1 \%$ because the volume fraction at positions $y \neq 0$ is double that of the volume fraction at $y=0$. A comparison of estimated and exact power saturation curves for a $25 \mathrm{~mm}$ long sample is shown in Fig. S6b; any visible differences disappear completely for sample lengths of $\sim 20 \mathrm{~mm}$.

We also neglect the (small) distortion of the mw field due to the sample's dielectric properties, which we expect to create a systematic error $<4 \%$ in $T_{1}$.

\section{The Einstein relation for charge and spin transport}

Figures $2 \mathrm{a}$ and $4 \mathrm{e}, \mathrm{f}$ in the main paper use the Einstein relation $\mu=e D /\left(k_{\mathrm{B}} T\right)=e \bar{R}^{2} /\left(k_{\mathrm{B}} T\right) \nu$ to estimate the charge's hopping distances $\bar{R}$ from the FET mobility $\mu$ and the charge's hopping rate $\nu$ (as determined from $T_{2}$ ). All quantities in this relation are therefore concerned with charge diffusion only, based on the previous conclusion that we can determine charge hopping rates from coherence times at low and intermediate temperatures. We would briefly like to comment here on whether a similar relation holds for spin transport, and possible implications for our analyses of motion frequencies and distances. 
Recent controversies whether such an Einstein relation also holds for spin diffusion stem from relating the charge diffusion constant $D$ to the spin diffusion length $\lambda_{\mathrm{S}}$ in the kinetic equation $\lambda_{\mathrm{S}}=\sqrt{D T_{1}} . \lambda_{\mathrm{S}}$ is typically determined from spin valves or spin-pumping devices with different organic layer thicknesses and $D$ is calculated from the charge carrier mobility in comparable device architectures (such as diodes) from the above Einstein relation ${ }^{9,10}$. This has lead to estimates for $T_{1}$ in organic semiconductors of $0.1-10 \mathrm{~ms}$, exceeding values from more direct measurements, such as in this work. Together with the absence of a Hanle effect in many such measurements, this has cast doubt on the validity of the Einstein relationship for spin transport.

We would like to note that our conclusions in the main paper are not directly affected by such controversies since we use the Einstein relation for charge transport only, where its applicability and limits for organic semiconductors are well established ${ }^{11,12}$. Nevertheless, a core hypothesis from spin valve and spin-pumping measurements is that distances between charge carriers become sufficiently small for exchange coupling to cause mutual spin-flips and to contribute significantly to spin diffusion ${ }^{13,14}$. This could result in much faster spin-diffusion compared to charge diffusion, resulting in overestimated spin lifetimes and possibly sufficiently rapid diffusion to prevent Hanle effect measurements.

Such an exchange-based spin transport mechanism could manifest itself in additional decoherence, by increasing spin "hopping rates" compared to charge hopping rates, and would complicate the calculation of charge hopping rates from $T_{2}$. First attempts to quantify such exchange-mediated transport in polymers predict an onset at carrier concentration $>10^{19}$ (Ref. [15]), exceeding those in our FI-ESR devices. This is also consistent with the independence of $T_{2}$ on the gate voltage in the EY-like relaxation regime (for instance in IDTBT above $140 \mathrm{~K}$ ): we expect any exchange-based mechanism to show a pronounced dependance on the average distance between polaron spins and therefore the carrier concentration. We can therefore exclude such contributions in our gated device architecture.

\section{Hall effect measurements in IDTBT}

In the main text, we exclude a momentum scattering Elliott-Yafet relaxation mechanism in IDTBT based on the absence of long-rage coherent charge transport. Evidence for this is provided by the temperature activated mobilities between 30-300 K (Fig. S3) and the absence of a Hall effect in IDTBT devices. This section will briefly present our attempts at measuring a Hall voltage for charge carriers in gated Hall bars.

In a semiclassical Boltzmann transport regime, the Hall voltage $V_{\mathrm{H}}$ is a measure of the transverse Lorentz force which a coherent wave packet with a defined wavevector experiences in a magnetic field $B$ perpendicular to the charge current $I$. In the so-called ideal Hall effect regime, the measured Hall coefficient $R_{\mathrm{H}}=V_{\mathrm{H}} t /(I B)$ is given by $R_{\mathrm{H}}=(e n)^{-1}$, where $n, t$ and $e$ are the carrier concentration, accumulation layer thickness and elementary charge, respectively. The Hall mobility $\mu_{\mathrm{H}}=\sigma R_{\mathrm{H}}$ then equals the actual charge carrier mobility $\mu=\sigma /(n e)$ where $\sigma$ denotes the conductivity. In contrast, if charge transport is dominated by incoherent hopping, Hall voltages tend to be much smaller and also exhibit sign anomalies ${ }^{16,17}$. In an intermediate transport regime with partially coherent charge transport - such as metallic domains separated by more disordered regions - one finds a so-called underdeveloped Hall effect with $\mu_{\mathrm{H}}<\mu^{18,19}$ and the ratio between the two mobilities $\gamma_{\mathrm{H}}=\mu_{\mathrm{H}} / \mu$ is termed the coherence factor. 
a

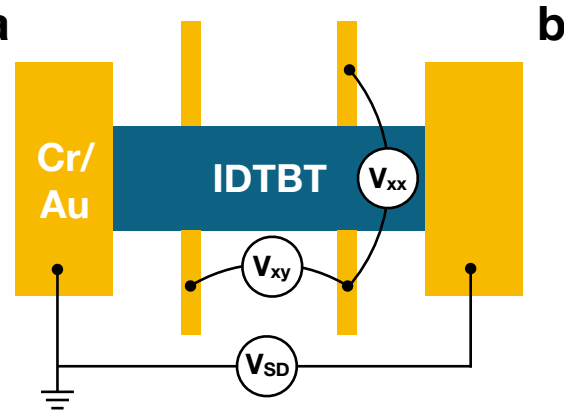

b

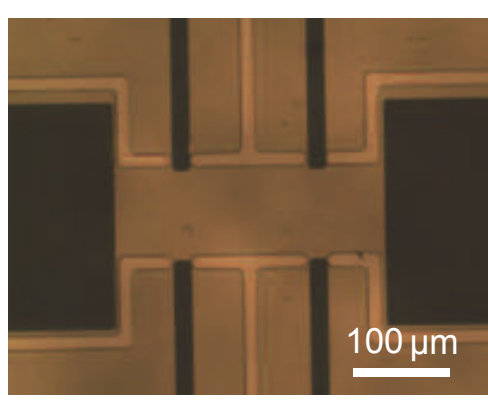

Figure S7: Schematic (a) and micrograph (b) of the Hall bar architecture.

We performed Hall effect measurements on IDTBT field-effect transistors in a Hall bar architecture with a channel length of $L=240 \mu \mathrm{m}$ and width of $W=80 \mu \mathrm{m}$ similar to Ref. [20]). Four $15 \mu \mathrm{m}$ wide Hall probes were positioned in between the source and drain electrodes, with a separation of $120 \mu \mathrm{m}$. The IDTBT film was lithographically patterned to a precise Hall bar geometry, following the procedure developed and published by our group in Refs. [21, 22], before spin-coating a $480 \mathrm{~nm}$ thick CYTOP dielectric layer and depositing an aluminium gate electrode by shadow mask evaporation (Fig. S7). IDTBT Hall bars show near-ideal transfer and output characteristics, even after the patterning process, and exhibit charge carrier mobilities around $1 \mathrm{~cm}^{2} \mathrm{~V}^{-1} \mathrm{~s}^{-1}$ from four-point-probe (4pp) and FET measurements (Fig. S8).

Hall effect measurements were carried out with an Oxford Instruments superconducting magnet where an external magnetic field $B$ was applied perpendicular to the sample plane. The longitudinal voltage $\Delta V_{x x}$ between adjacent probes, and the transverse voltage $\Delta V_{x y}$ between opposite probes were recorded for a constant source-drain voltage, $V_{\mathrm{SD}}$, while $B$ was ramped between $8 \mathrm{~T}$ and $-8 \mathrm{~T}$ with a sweep rate of $0.2 \mathrm{~T} \mathrm{~min}^{-1}$. The devices were operated in the linear regime during the Hall effect measurements, with an applied gate voltage of $V_{\mathrm{G}}=-40 \mathrm{~V}$ and a source-drain voltage of $V_{\mathrm{SD}}=-5 \mathrm{~V}$. Figure $\mathrm{S} 9$ shows the measured magnetic field, source drain current $I_{\mathrm{SD}}$ and transverse voltage $V_{x y}$ as a function of time. Note that $V_{x y}$ exhibits a constant offset, presumably due to a misalignment of the transverse probes with respect to the patterned Hall bar. Such an offset can be estimated from $V_{x y}^{\text {offset }}=\frac{I}{\sigma} \frac{\Delta x_{14}}{W t}$ where $\Delta x_{14}$ is the longitudinal misalignment between the two opposite probes. This gives a misalignment on the order of $\sim 1 \mu \mathrm{m}$, consistent with the resolution of our patterning process.
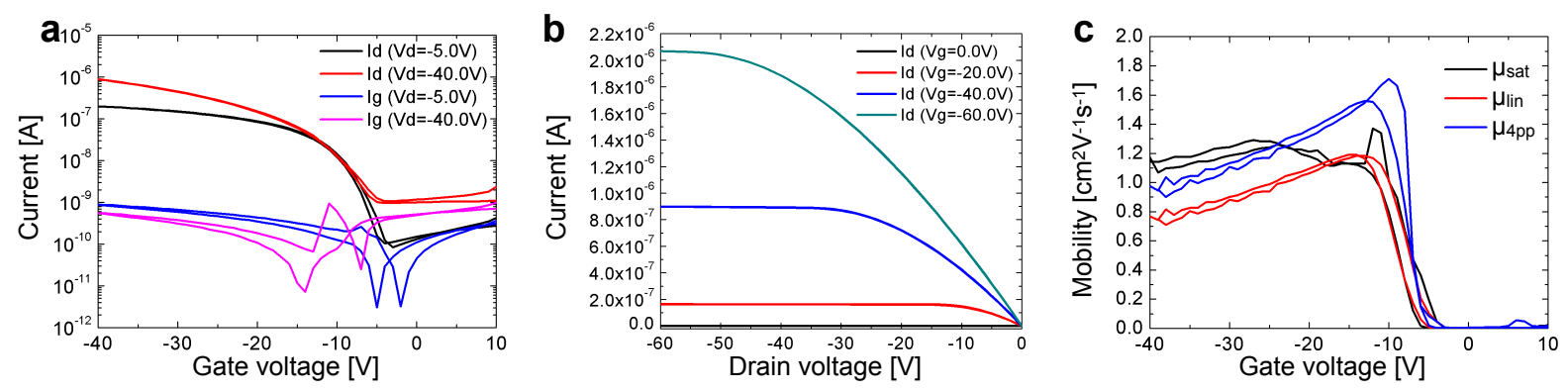

Figure S8: (a, b) Transfer and output characteristics of a representative IDTBT Hall bar device and (c) extracted gate voltage dependence of the linear, saturation and four-point-probe (4pp) mobilities. 
The expected Hall voltage in case of an ideal Hall effect can be estimated from the field-effect mobility to be

$$
V_{\mathrm{H}}=\frac{R_{\mathrm{H}} I_{\mathrm{SD}}}{t} B=\frac{\mu W V_{\mathrm{D}}}{L} B \approx 1.3 \mathrm{mV}
$$

for a magnetic field of $B=8 \mathrm{~T}$. From Fig. S9, it is evident that no Hall voltage could be observed. With the noise level $\sim 150 \mu \mathrm{V}$ in our measurements, we can give an upper limit for the coherence factor in IDTBT of $\gamma_{\mathrm{H}} \lesssim 0.06$ which rules out the formation of extended Bloch states.

Finally, we would like to note that even where a Hall effect has been observed in polymeric semiconductors, it is often underdeveloped, indicating an interplay of coherence and localization effects $^{20,23-25}$ which cannot be explained by a pure band model but supports our interpretation of spin lifetimes in terms of a transient localization scenario. This is the case for instance in pBTTT where Hall voltages could be detected in highly doped thin films ${ }^{20}$.



Figure S9: Magnetic field, drain current and transverse voltage $V_{x y}$ as a function of time during a Hall effect measurement on an IDTBT device. No change in the transverse voltage is visible while sweeping the magnetic field.

\section{DFT calculation of spin admixtures}

A major spin relaxation mechanism in a general semi-conductor is scattering between states of mixed spin, due to spin-orbit coupling (SOC). This is discussed in the main text in the context of the Elliott-Yafet ${ }^{26,27}$ (EY) spin relaxation mechanism. Since molecular SOC also mixes spins, ${ }^{28}$ an EY-like mechanism acts in a molecular semi-conductor (see main text).

We quantify spin-mixing by the dimensionless spin admixture parameter $\gamma$. This parameter is the first order correction from perturbation theory to the SOC-free Hamiltonian. It is calculated as the deviation from the norm of unperturbed, SOC-free, pure spin eigenstates to that of perturbed, mixed eigenstates constructed in the basis of the pure spin eigenstates. We follow the procedure published by $\mathrm{Yu}^{29,30}$ to calculate this quantity from a density functional theory (DFT) wavefunction, with several major improvements. 
First, we generalize this technique from the original restricted open shell Hartree-Fock (ROHF) approximation to the unrestricted Hartree-Fock (UHF) approximation. This eliminates severe variational restrictions on the molecular groundstate. Next, instead of the original semi-empirical approximation of the SOC matrix elements, we calculate these from first-principles using the zeroth order regular approximation ${ }^{31}$ (ZORA) to the fully-relativistic Hamiltonian, resulting in significantly improved quality. This improvement also lifts the restriction of the original formulation to a minimal atomic orbital basis sets, allowing us to converge $\gamma$ with respect to the basis set. The minimally augmented, single-zeta, polarized SARC basis set ${ }^{32}$ (ma-zora-def2-svp), which has been recontracted for ZORA, was found sufficient for this study. The minimal augmentation (diffuse) functions were removed from the carbon atoms in order to eliminate linear dependencies, but otherwise, this basis set was used as published. The original calculations ${ }^{30}$ were also significantly affected by electron delocalization error stemming from the employed semi-local exchange-correlation functional. We eliminate $25 \%$ of this error by performing all calculations using the hybrid PBE0 ${ }^{33}$ exchange-correlation functional.

In practice, the calculations are performed starting from a structure predicted by DFT, or as needed by e.g. force fields in the theoretically predicted morphologies. Then a charge is introduced, and the size of the structural sub-unit chosen such that $\gamma$ no longer varies with it. This system is calculated with a multiplicity of 2 , and the final $\gamma$ is calculated from the resulting DFT wavefunction. All DFT calculations were performed with version 6.5 of the NWChem computational chemistry package ${ }^{34}$.

\section{Estimate of intra-site relaxation in IDTBT}

Besides being the source of rapid charge density dynamics, vibrational modes can directly couple to the spin by modulating HFI- and g-tensors the spin's rest frame. Such spin-vibron coupling is known in literature as intra-site relaxation and takes place between hopping events while the wave-function remains localized at a single site ${ }^{35-37}$. In the section, we will give a brief estimate of the expected relaxation times in this static localization picture, based on the Redfield equations and the simulated frequencies and amplitudes of torsional modes from Fig. 3c,d in the main paper.

To this end, we consider the modulation of HFI- and g-tensors $\mathbf{g}$ and $\mathbf{A}$, respectively, with vibrational modes. Torsional modes will be most effective at driving intra-site relaxation; first, because their low frequencies $\tau_{\mathrm{c}}^{-1}>\omega_{\mathrm{L}}$ come closest to the Zeeman splitting energy $\hbar \omega_{\mathrm{L}}$, and second, because a rotation of the corresponding coupling tensors translates to large changes in the local effective fields. The isotropic parts of the tensors will remain constant under rotations and the spin lifetimes therefore only depend on the anisotropic components $\Delta \mathbf{g}=\mathbf{g}-g_{\text {iso }} 1$ and $\Delta \mathbf{A}=\mathbf{A}-A_{\text {iso }}$ 1. For a uniformly rotating segment with $\tau_{\mathrm{c}}^{-1}>\omega_{\mathrm{L}}$, one obtains with the Redfield theory $^{38}$ :

$$
\begin{aligned}
T_{1}^{-1}=T_{2}^{-1}= & \left(\frac{\mu_{\mathrm{B}} B_{0}}{\hbar}\right)^{2}(\Delta \mathbf{g}: \Delta \mathbf{g}) \frac{1}{10} \frac{\tau_{\mathrm{c}}}{1+\omega_{L}^{2} \tau_{\mathrm{c}}^{2}} \\
& +\sum_{k} I_{k}\left(I_{k}+1\right)\left(\Delta \mathbf{A}_{k}: \Delta \mathbf{A}_{k}\right) \frac{7}{60} \frac{\tau_{\mathrm{c}}}{1+\omega_{L}^{2} \tau_{\mathrm{c}}^{2}}
\end{aligned}
$$

where $B_{0} \simeq 335 \mathrm{mT}$ denotes the external magnetic field, $\mathbf{x}: \mathbf{y}$ indicates the component-wise inner product of two matrices $\mathbf{x}$ and $\mathbf{y}$, and the summation runs over all nuclear spins $I_{k}$ with HFI tensors $\mathbf{A}_{k}$ in angular frequency units. With a measured difference of $200 \mathrm{ppm}$ between in-plane 
and out-of-plane components of the g-tensor in IDTBT, we estimate that $(\Delta \mathbf{g}: \Delta \mathbf{g}) \simeq(200 \mathrm{ppm})^{2}$. We can get an upper bound for the contribution of anisotropic HFIs by assuming that the Gaussian broadening of $\delta B_{\mathrm{rms}} \simeq 0.34 \mathrm{mT}$ at $5 \mathrm{~K}$ is dominated by such interactions, such that the second term in Eq. (S23) becomes $\gamma_{\mathrm{e}}^{2} \delta B_{\mathrm{rms}}^{2} \tau_{\mathrm{c}} /\left(1+\omega_{L}^{2} \tau_{\mathrm{c}}^{2}\right)$.

With the above considerations, the relaxation time from the lowest frequency torsional modes at $\tau_{\mathrm{c}}^{-1} \simeq 0.1 \mathrm{THz}$ can then be estimated to be $T_{1}^{\mathrm{vib}}=T_{2}^{\mathrm{vib}}=7 \mu$ s for uniform rotations, $S=0$, which significantly exceeds experimental values. Rescaling the amplitudes in Eq. (S23) for $S \approx 0.9$, one obtains lifetimes on the order of several $100 \mu \mathrm{s}$ for the lowest frequency modes (and much longer ones for higher frequencies). This significantly exceeds experimental values and suggests that despite exhibiting the correct temperature activation, the direct coupling of the spin to such torsional modes in a static localization picture cannot explain the observed relaxation times.

\section{GIWAXS measurements}

Grazing Incidence Wide-Angle X-ray Scattering (GIWAXS) measurements were performed at the SAXS/WAXS beamline at the Australian Synchrotron ${ }^{39}$. $15 \mathrm{keV}$ photons were used with 2dimensional scattering patterns recorded on a Dectris Pilatus 1M detector. Images were acquired at an incident angle close to the critical angle, chosen from a series of images taken with incident $\mathrm{X}$-ray angles varying from $0.02^{\circ}$ to $0.15^{\circ}$ in steps of $0.01^{\circ}$ with the chosen image showing the highest scattering intensity. The X-ray exposure time was $1 \mathrm{sec}$ such that no film damage was identified. The sample-to-detector distance was calibrated using a silver behenate sample. The results were analyzed by an altered version of the NIKA $2 \mathrm{D}$ software $^{40}$ based on IgorPro.
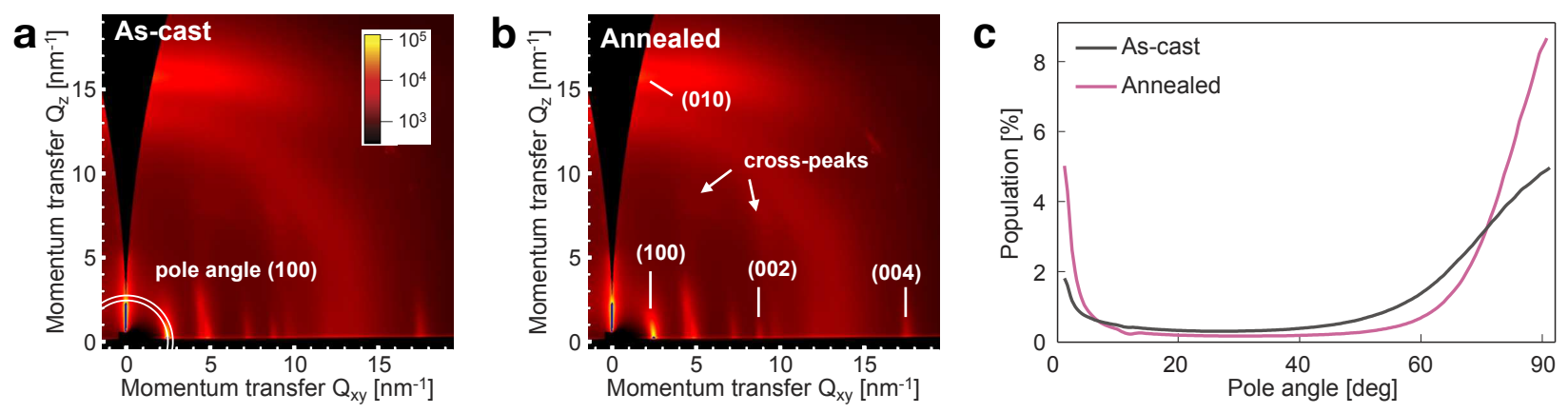

Figure S10: (a, b) GIWAXS patterns of as-cast and annealed p(NDI2OD-T2) films. (c) Crystallite orientation distribution profiles (COD) extracted from the circular line cut across the (100) diffraction indicated in Fig. 5a. 0 deg denotes the direction perpendicular to the substrate. Annealed p(NDI2ODT2) forms more face-on crystallites, as evidenced by the higher population for pole angles $>70$ deg (data close to 0 deg are affected by 'specular spray' and may not reflect scattering from crystallites).

The resulting scattering patterns for as-cast and annealed $\mathrm{p}(\mathrm{NDI} 2 \mathrm{O}-\mathrm{T} 2)$ films are shown in Fig. S10a,b. Peak assignments are based on previous reports ${ }^{41,42}$ and respective stacking distances and coherence lengths are given in Table S1. The GIWAXS patterns of annealed films show emerging mixed-index peaks indicative of enhanced three-dimensional order and a narrower distribution of crystallite orientations. The narrowing of diffraction peaks upon annealing indicates an increase in the average crystallite volume by a factor of 4 and the average lamellar area by a factor of 2 . On the other hand, the lattice spacings are only marginally affected. 
Crystallite orientation distribution profiles (COD) were extracted from a circular line cut across the (100) diffraction and are shown in Fig. S10c. The angular populations of backbone tilt angles were calculated by normalizing the measured diffraction intensity by the integrated total intensity within the pole angle range of interest, assuming that crystallites from the same crystallographic direction at different pole angles are formed with similar crystallite thickness and lattice disorder.

\begin{tabular}{lcccccc}
\hline & \multicolumn{2}{c}{ Lamellar (IP) } & \multicolumn{2}{c}{ Backbone (IP) } & \multicolumn{2}{c}{$\pi$ - $\pi$ stacking (OOP) } \\
& $\begin{array}{c}\text { Spacing } \\
{[\mathrm{nm}]}\end{array}$ & $\begin{array}{c}\text { Coh. length } \\
{[\mathrm{nm}]}\end{array}$ & $\begin{array}{c}\text { Spacing } \\
{[\mathrm{nm}]}\end{array}$ & $\begin{array}{c}\text { Coh. length } \\
{[\mathrm{nm}]}\end{array}$ & $\begin{array}{c}\text { Spacing } \\
{[\mathrm{nm}]}\end{array}$ & $\begin{array}{c}\text { Coh. length } \\
{[\mathrm{nm}]}\end{array}$ \\
\hline As-cast & 2.45 & 20.13 & 1.40 & 16.48 & 0.387 & 2.12 \\
Annealed & 2.53 & 38.06 & 1.42 & 20.73 & 0.382 & 3.49 \\
\hline
\end{tabular}

Table S1: In-plane (IP) and out-of-plane (OOP) stacking distances and coherence lengths for as-cast and annealed $\mathrm{p}$ (NDI2O-T2) films. Lamellar spacing is determined from the (100), backbone spacing from the (002), and $\pi$-spacing from the (010) peak position. Coherence lengths are determined from the respective peak widths by the Scherrer equation.

\section{Raman measurements}

The Raman data were taken with a Jobin Yvon Horiba T64000 Triple Raman Spectrometer on $\mathrm{p}$ (NDI2OD-T2) thin films, spin-coated on silicon substrates under the same conditions as FI-ESR devices. Scans were taken in the single stage mode with $532 \mathrm{~nm}$ laser excitation. Both spectra consist of two scans each with a 5 seconds exposure time.

The resulting spectra are shown in Fig. S11a. From Ref. [43], we can assign the peaks at $1407 \mathrm{~cm}^{-1}, 1434 \mathrm{~cm}^{-1}$, and $1463 \mathrm{~cm}^{-1}$ to $\mathrm{C}=\mathrm{C} / \mathrm{C}-\mathrm{C}$ bond stretching/shrinking modes on NDI2OD, the whole NDI2OD-T2 monomer, and on the thiophene units, respectively. The difference in relative peak amplitudes can be traced back to a narrowing of the carbon-carbon stretching resonances at $1400-1500 \mathrm{~cm}^{-1}$ for the annealed film while relative peak areas remain unaffected (see Fig. S11b,c). This is consistent with a larger crystallite size and less orientational disorder in the annealed samples.
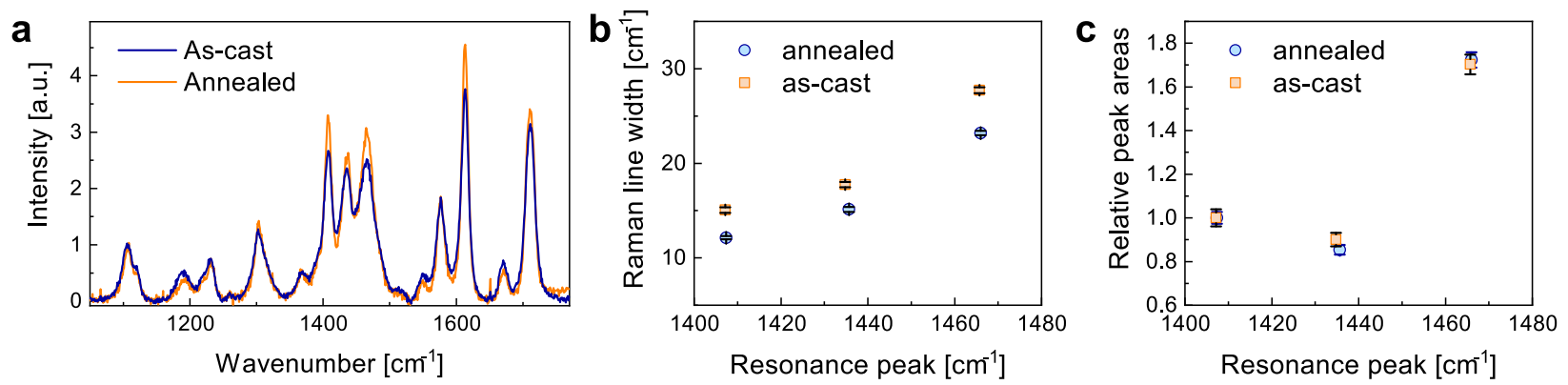

Figure S11: (a) Normalized Raman spectra of as-cast and annealed p(NDI2O-T2) films (532 nm excitation). (b) Linewidths of carbon-carbon stretching modes at $1407 \mathrm{~cm}^{-1}$ (localized on the NDI unit), $1435 \mathrm{~cm}^{-1}$ (delocalized over the whole monomer) and $1466 \mathrm{~cm}^{-1}$ (localized to the thiophene units). (c) Relative peak areas, normalized to the $1407 \mathrm{~cm}^{-1}$ resonance, of the same resonances from (b). Changes in relative peak areas upon annealing are insignificant. 


\section{Torsional autocorrelation functions}

The simulated autocorrelation functions of a torsional angle $\varphi$ for both p(NDI2OD-T2) and IDTBT have been calculated as:

$$
\langle\cos (\varphi(\tau)-\varphi(0))\rangle=\sum_{n=0}^{M} \sum_{i=0}^{N} \sum_{\tau=0}^{N-i}\left[\cos \left(\alpha_{n}(i)\right) \cos \left(\alpha_{n}(i+\tau)\right)+\sin \left(\alpha_{n}(i)\right) \sin \left(\alpha_{n}(i+\tau)\right)\right]
$$

where $M$ is the total number of IDT-BT torsions within the 10 pentamers of the IDTBT amorphous morphology or NDI-thiophene and thiophene-thiophene torsion angles within the 24 dodecamers of a $\mathrm{p}(\mathrm{NDI} 2 \mathrm{OD}-\mathrm{T} 2)$ morphology and $N$ is the number of molecular dynamics (MD) snapshots. From the autocorrelation functions, we have obtained the power spectrum from fast Fourier transform analysis (see Fig. S12).
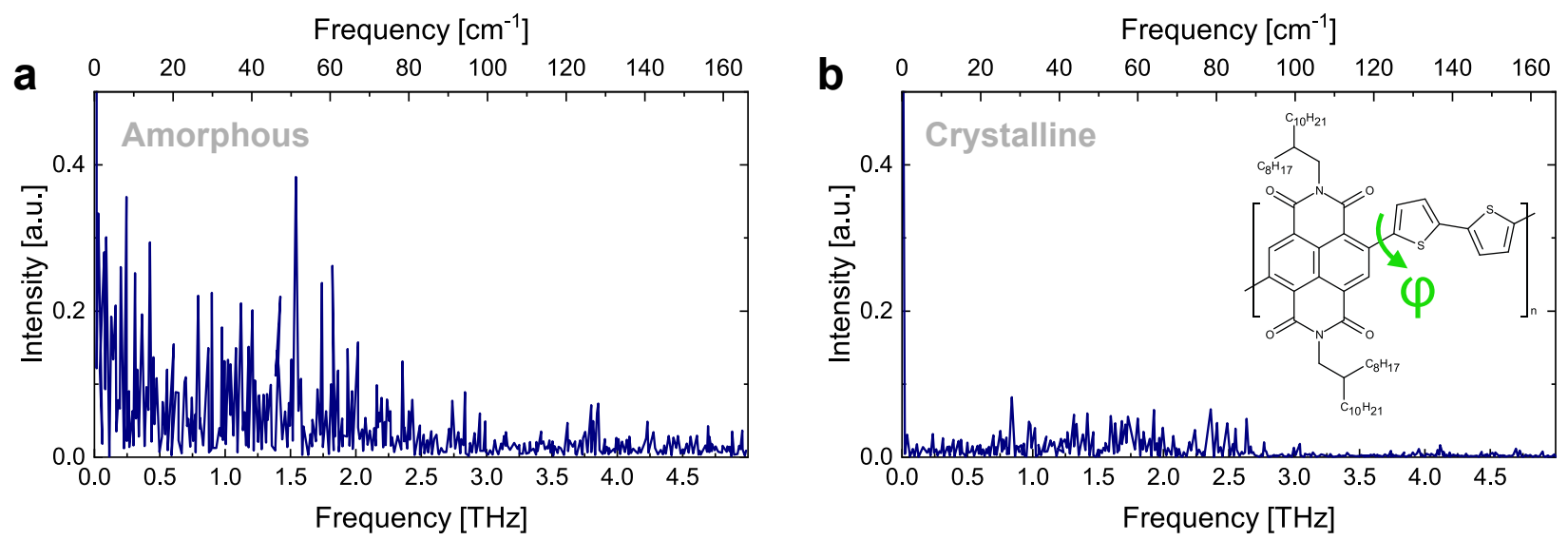

Figure S12: Simulated autocorrelation functions of the NDI-T torsional angle $\varphi$ (see inset) for (a) amorphous and (b) crystalline phases published in Ref. [44]. The crystalline phase shows reduced amplitudes as well as a narrowing and shift of modes to higher frequencies.

The data used for these simulations comes from a conformational analysis of crystalline and amorphous phases of $\mathrm{p}(\mathrm{NDI} 2 \mathrm{OD}-\mathrm{T} 2)$ generated at the MD level. In practice, we first built, using periodic boundary conditions, a large crystalline phase by replicating the simulated crystalline unit cell published in Ref. [44]. The size of the crystalline system studied here corresponds to three layers of eight $\pi$-stacked dodecamers. Then, a $2.5 \mathrm{~ns}$-long MD run (NPT, $P=1 \mathrm{~atm}, T=298 \mathrm{~K}$ ) is performed to equilibrate the system followed by a 200 ps-long MD (NPT, $P=1 \mathrm{~atm}, T=298 \mathrm{~K}$ ) from which simulated torsion angles were extracted every $100 \mathrm{fs}$ for each NDI-T and T-T angles. The torsion angles used to generate the autocorrelation functions for the amorphous phase also come from a $200 \mathrm{ps}$-long MD (NPT, $P=1 \mathrm{~atm}, T=298 \mathrm{~K})$ from which data were extracted every $100 \mathrm{fs}$ for each NDI-T and T-T angles. To generate an equilibrated amorphous phase, we used the following protocol: We first randomly oriented 24 dodecamers of $\mathrm{p}$ (NDI2OD-T2) in a large unit cell $(300 \AA \times 300 \AA \times 300 \AA)$ and subjected it to a 500 ps-long MD run at a high temperature (NVT; $T=1000 \mathrm{~K})$ while keeping the density low $\left(\sim 0.02 \mathrm{~g} \mathrm{~cm}^{-3}\right)$ to favor a random spatial distribution of the oligomers. Then, 4 successive 500 ps-long MD runs (NPT, $P=1 \mathrm{~atm}$ ) are performed at decreasing temperatures $(1000 \mathrm{~K}, 500 \mathrm{~K}, 400 \mathrm{~K}, 350 \mathrm{~K})$ followed by a $2.5 \mathrm{~ns}-$ long MD simulation $(\mathrm{NPT} ; P=1 \mathrm{~atm}, T=298 \mathrm{~K})$. 
All MM/MD calculations have been performed with the Materials Studio (MS) 6.0 package using a force-field derived from Dreiding ${ }^{45}$ in which the torsion potentials between adjacent subunits and the torsions between the conjugated cores and the alkyl chains have been benchmarked against Density Functional Theory (DFT) calculations using the B3LYP functional and the cc-pvtz basis set $^{46}$. The atomic charges have been obtained by fitting the electrostatic potential (ESP charges ${ }^{47}$ ) calculated at the B3LYP/cc-pvtz level on an isolated dimer.

\section{Dynamical disorder in IDTBT}

Using the DFT method (B3LYP functional and 6-311+g(d,p) basis set), we optimized an IDTBT monomer in vacuum (see Fig. S13, side-chains reduced to methyls), and calculated vibrational frequencies in its respective geometry. Since IDTBT is a hole-conducting polymer, we investigate two cases: a neutral state (total charge $q=0)$ and a cationic state $(q=+1)$. In a neutral state, the lowest frequency is $\omega_{1}^{(0)}=1.9 \times 10^{11} \mathrm{~Hz}$ and corresponds to oscillations around the dihedral angle $\phi$, shown in Fig. 1. The following two modes are $\omega_{2}^{(0)}=6.4 \times 10^{11} \mathrm{~Hz}$ and $\omega_{3}^{(0)}=1.4 \times 10^{12} \mathrm{~Hz}$, and correspond to the out-of-plane oscillations. Similarly, in a cation state, the frequency of the dihedral mode $\phi$ is twice the one in a ground state, $\omega_{1}^{(0)}=3.5 \times 10^{11} \mathrm{~Hz}$, which is consistent with higher planarity of the backbone due to charging. The following two out-of-plane modes have frequencies

$\omega_{2}^{(0)}=6.1 \times 10^{11} \mathrm{~Hz}$ and $\omega_{3}^{(0)}=1.3 \times 10^{12} \mathrm{~Hz}$. In both states, the calculated dihedral frequencies are within the anticipated range.

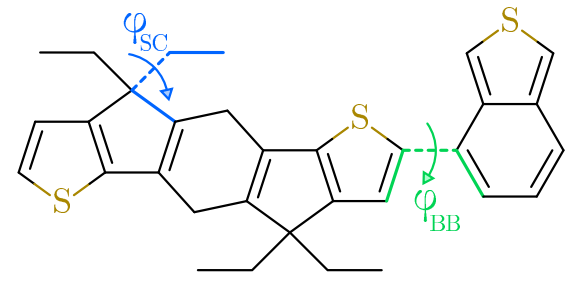

Figure S13: Structure of the IDTBT force field and re-parameterized dihedrals: $\phi_{\mathrm{BB}}$ connects IDT and BTZ units in a backbone, while $\phi_{\mathrm{SC}}$ connects side-chains to a backbone via a bridging carbon. Respective PES scans are shown in Fig. S14.

To simulate torsional dynamics, we first constructed a unit cell and built a super cell of 8 lamellae $\times 16$ chains, i.e., 128 tetramers in total. The supercell was equilibrated for $1 \mathrm{~ns}$ in the isothermal-isobaric (NTP) ensemble at $100 \mathrm{~K}$. These trajectories, 1000 snapshots each, were used for further analysis.

In order to simulate morphologies of the polymer, we developed an atomistic force field. The chemical structure of an IDTBT monomer used to parametrize the force field is shown in Fig. S13. A monomer with side-chains reduced to ethyls is optimized using the B3LYP/6- 311g(d,p) as a functional and a basis set. Partial charges are obtained using the Merz-Kollman method ${ }^{47,48}$.

All Lennard-Jones parameters for non-bonded interactions are taken from the OPLS-AA force field ${ }^{49-52}$, while differentiating between conjugated fragments and hydrocarbons.

Values for bonds and angles are taken from the DFT-optimized geometry, while most spring and dihedral constants are taken from the OPLS-AA ${ }^{49-52}$ force field. Two dihedrals, $\phi_{\mathrm{BB}}$ and $\phi_{\mathrm{SC}}$ (Fig. S13), were re-parametrized by matching first-principle and molecular dynamics (MD) force 



Figure S14: Re-parametrization of the potential energies for the backbone dihedral angle $\phi_{\mathrm{BB}}(\mathbf{a})$ and the dihedral angle $\phi_{\mathrm{SC}}(\mathbf{b})$, as defined in Fig. S13. Blue markers indicate the QM values, the red curve is from MD with an optimized force field, the green curve denotes the difference between the two.

field potential energy surfaces (PES). If $q$ is the degree of freedom of interest, constrained geometry optimizations must be performed using both first-principles and the force-field levels, yielding the total energies $U_{\mathrm{DFT}}(q)$ and $U_{\mathrm{FF}}^{\text {initial }}(q)$, respectively. The missing force-field $(\mathrm{FF})$ terms are then fitted to this difference using a prescribed functional form. Finally, the constrained geometry optimizations are repeated on the force-field level including the obtained potential and $U_{\mathrm{FF}}^{\mathrm{final}}(q)$ is compared to the first-principles scan.

For a potential corresponding to the dihedral angle $\phi_{\mathrm{BB}}$ we used the Ryckaert-Belleman form

$$
V_{\mathrm{RB}}\left(\phi_{i j k l}\right)=\sum_{n=0}^{5} C_{n} \cos ^{n}\left(\phi_{i j k l}\right),
$$

while for a potential corresponding to the dihedral angle $\phi_{\mathrm{SC}}$ we used a truncated Fourier series

$$
\left.V_{a}\left(\phi_{i j k l}\right)=\sum_{n=0}^{N} C_{n} \cos \left(n \phi_{i j k l}-\phi_{i j k l}^{(n)}\right)\right)
$$

to account for a more complex, asymmetric PES shape, arising from a presence of bridging carbons. An upper cut-off at $N=6$ was sufficient to reproduce the quantum mechanical (QM) curve. The fits are shown in Fig. S14.

We used the velocity rescaling thermostat ${ }^{53}$, the Berendsen barostat ${ }^{54}$, while electrostatics and van der Waals interactions were treated with a cut-off at $r_{\mathrm{c}}=1.2 \mathrm{~nm}$.

Quantum corrections are taken into account by introducing an effective temperature. This is achieved by replacing the classical Boltzmann distribution with a suitable quantum equivalent.

In order to introduce quantum corrections, a vibronic density of states (DOS) is required. To make calculations feasible, we calculated a vibronic DOS for an IDTBT tetramer in vacuum, with side-chains reduced to $\mathrm{CH}_{3}$ groups. For the same reason, we used a PM3 semi-empirical method to optimize the geometry and to calculate frequencies in this geometry ${ }^{55}$. A vibronic DOS is typically normalized as

$$
\int_{0}^{\infty} g(\omega) \mathrm{d} \omega=3 N-6
$$


where $3 N$ is the number of DOFs, $g(\omega)$ is a simulated vibronic density of states. The resulting spectrum is shown in Fig. S15 and is comparable to measured IR and Raman spectra of similar conjugated polymers ${ }^{56,57}$.

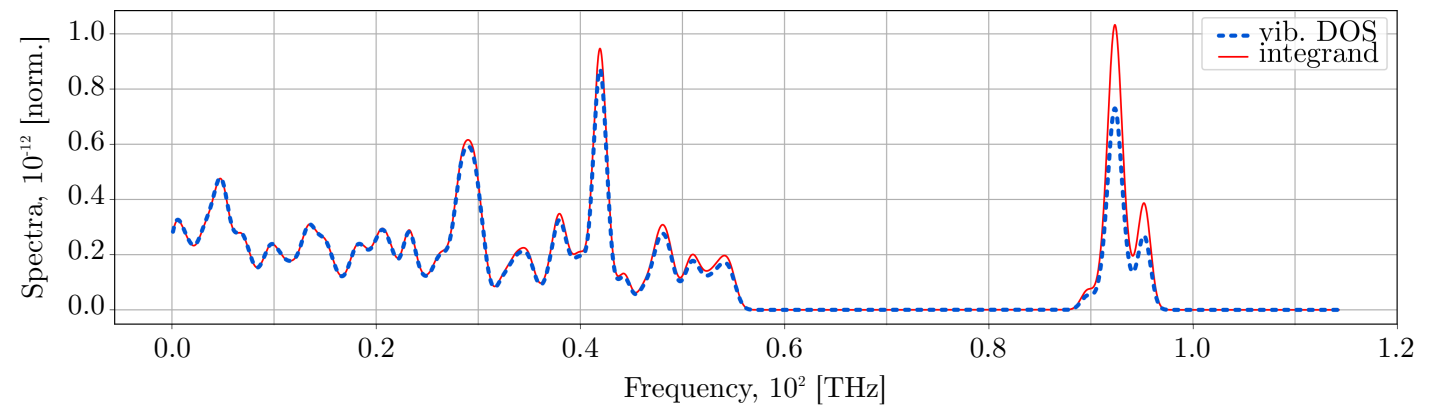

Figure S15: Simulated vibronic DOS $g(\omega)$ of an IDTBT tetramer and the integrand $g(\omega) W\left(\omega, T_{\mathrm{QM}}\right)$ used in Eq. (S28), multiplied by a factor 0.026 for comparison.

Quantum corrections are introduced by re-weighting the system's vibrational DOS. Namely, the following equation holds ${ }^{58}$

$$
\int_{0}^{\infty} g(\omega) W\left(\omega, T_{\mathrm{QM}}\right) \hbar \omega \mathrm{d} \omega=3 N k_{\mathrm{B}} T_{\mathrm{MD}}
$$

where $W\left(\omega, T_{\mathrm{QM}}\right)$ is a sum of a Bose-Einstein distribution and a zero-point energy

$$
W\left(\omega, T_{\mathrm{QM}}\right)=\frac{1}{\exp \left(\frac{\hbar \omega}{k_{\mathrm{B}} T_{\mathrm{QM}}}\right)-1}+\frac{1}{2},
$$

as opposed to the Boltzmann distribution. $T_{\mathrm{MD}}$ and $T_{\mathrm{QM}}$ denote the temperatures from molecular dynamics and the effective "quantum" temperature of the system, respectively. Provided the calculated vibronic DOS above, one can invert Eq. (S28) and obtain $T_{\mathrm{QM}}$.

Figure S16 shows the calculated MD-to-QM temperature mapping, and that effective temperatures are notably smaller than the ones used in MD. Within the simulated range, $T_{\mathrm{MD}}=200 \mathrm{~K}$ corresponds to $T_{\mathrm{QM}}=150 \mathrm{~K}$, i.e., the reduction factor is close to $3 / 4$. We then fitted the blue curve with a polynomial function, and applied it to the simulated dynamic order parameter curves. This effectively shifted the curves (and the transition temperature) to lower temperatures. The final result is reported in the main text, where $T$ refers to the effective, "quantum" temperature.

Molecular dynamics simulations were performed using the GROMACS simulation package ${ }^{59}$, while first-principles calculations were performed in the Gaussian G09 simulations package. Dynamic order parameters $S$ are calculated using in-house python scripts, based on numpy and scipy python libraries. Figures are produced using the matplotlib python library and the inskcape graphics package. Snapshot figures are produced using the VMD program ${ }^{60}$. 


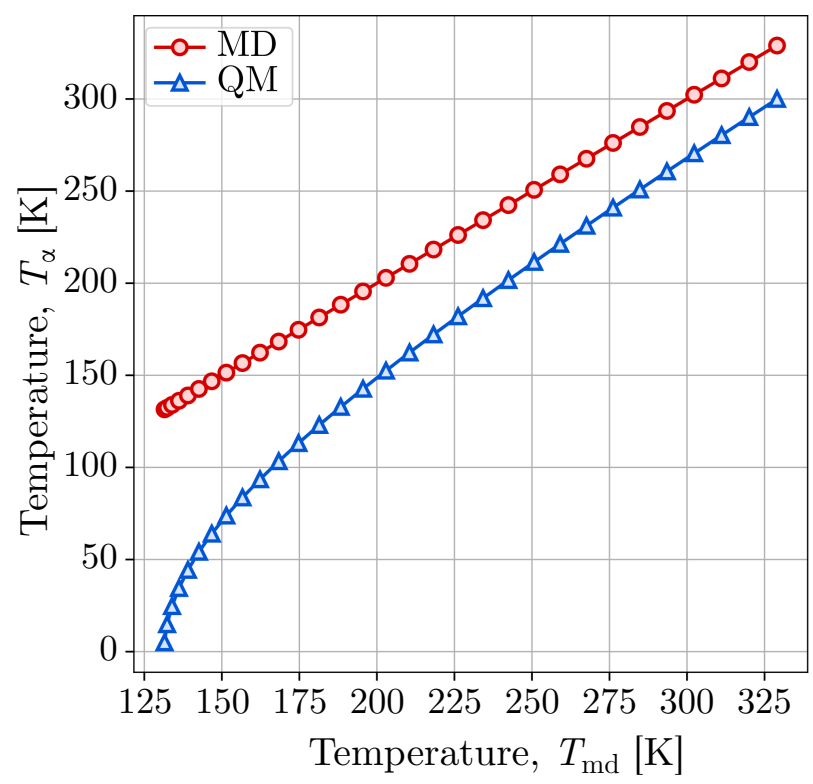

Figure S16: Relation between MD (classical) and QM (real) temperatures.

\section{Supplementary References}

1. Bao, Z. \& Locklin, J. Organic Field-Effect Transistors Is B N: 978-1-4200-0801-2 (CRC Press, 2007).

2. Brütting, W. \& Adachi, C. Physics of Organic Semiconductors Is B N: 3-527-65496-8 (John Wiley \& Sons, 2012).

3. Eaton, G. R., Eaton, S. S., Barr, D. P. \& Weber, R. T. Quantitative EPR is B N: 978-3-21192947-6 (Springer Vienna, Vienna, 2010).

4. Slichter, C. P. Principles of Magnetic Resonance IS B N: 3-662-12784-9 (Springer Science \& Business Media, 2013).

5. Hyde, J. S., Pasenkiewicz-Gierula, M., Jesmanowicz, A. \& Antholine, W. E. Pseudo Field Modulation in EPR Spectroscopy. Appl. Magn. Reson. 1, 483-496 (1990).

6. Stoll, S. \& Schweiger, A. EasySpin, a Comprehensive Software Package for Spectral Simulation and Analysis in EPR. J. Magn. Reson. 178, 42-55 (2006).

7. Korb, J. P. \& Maruani, J. Measurement of Spin-Spin and Spin-Lattice Relaxation Rates for Inhomogeneously Broadened Lines. I. Rapid Spectral Diffusion Case. J. Magn. Reson. 37, 331-348 (1980).

8. Portis, A. M. Electronic Structure of F Centers: Saturation of the Electron Spin Resonance. Phys. Rev. 91, 1071-1078. Is s N: 0031-899X (1953).

9. Xiong, Z. H., Wu, D., Valy Vardeny, Z. \& Shi, J. Giant Magnetoresistance in Organic SpinValves. Nature 427, 821-824. IS S N: 1476-4687 (2004).

10. Watanabe, S. et al. Polaron Spin Current Transport in Organic Semiconductors. Nat. Phys. 10, 308-313 (2014). 
11. Li, L., Lu, N., Liu, M. \& Bässler, H. General Einstein Relation Model in Disordered Organic Semiconductors under Quasiequilibrium. Phys. Rev. B 90. Is s n: 1098-0121, 1550-235X (2014).

12. Wetzelaer, G. A. H., Koster, L. J. A. \& Blom, P. W. M. Validity of the Einstein Relation in Disordered Organic Semiconductors. Phys. Rev. Lett. 107, 066605 (2011).

13. Yu, Z. G. Suppression of the Hanle Effect in Organic Spintronic Devices. Phys. Rev. Lett. 111, 016601 (2013).

14. Jiang, S. W. et al. Exchange-Dominated Pure Spin Current Transport in Alq3 Molecules. Phys. Rev. Lett. 115, 086601 (2015).

15. Wang, S.-J. et al. Long Spin Diffusion Lengths in Doped Conjugated Polymers Due to Enhanced Exchange Coupling. Nat. Electron. 2, 98-107 (2019).

16. Friedman, L. Hall Conductivity of Amorphous Semiconductors in the Random Phase Model. Journal of Non-Crystalline Solids 6, 329-341. Is S N: 0022-3093 (1971).

17. Klein, R. S. Investigation of the Hall Effect in Impurity-Hopping Conduction. Phys. Rev. B 31, 2014-2021 (1985).

18. Seeger, K., Gill, W. D., Clarke, T. C. \& Street, G. B. Conductivity and Hall Effect Measurements in Doped Polyacetylene. Solid State Commun. 28, 873-878 (1978).

19. Shacklette, L. W., Chance, R. R., Ivory, D. M., Miller, G. G. \& Baughman, R. H. Electrical and Optical Properties of Highly Conducting Charge-Transfer Complexes of Poly(p-Phenylene). Synthetic Metals 1, 307-320. Is N : 0379-6779 (1980).

20. Kang, K. et al. 2D Coherent Charge Transport in Highly Ordered Conducting Polymers Doped by Solid State Diffusion. Nat. Mater. 15, 896-902 (2016).

21. Chang, J.-F. \& Sirringhaus, H. Patterning of Solution-Processed Semiconducting Polymers in High-Mobility Thin-Film Transistors by Physical Delamination. Adv. Mater. 21, 2530-2535. IS S N : 1521-4095 (2009).

22. Chang, J. F. et al. Hall-Effect Measurements Probing the Degree of Charge-Carrier Delocalization in Solution-Processed Crystalline Molecular Semiconductors. Phys. Rev. Lett. 107, 066601 (2011).

23. Wang, S., Ha, M., Manno, M., Daniel Frisbie, C. \& Leighton, C. Hopping Transport and the Hall Effect near the Insulator-Metal Transition in Electrochemically Gated Poly(3-Hexylthiophene) Transistors. Nat. Commun. 3, 1210 (2012).

24. Yi, H. T., Gartstein, Y. N. \& Podzorov, V. Charge Carrier Coherence and Hall Effect in Organic Semiconductors. Sci. Rep. 6, 23650 (2016).

25. Anderson, M. et al. Displacement of Polarons by Vibrational Modes in Doped Conjugated Polymers. Phys. Rev. Mater. 1, 055604-9 (2017).

26. Elliott, R. J. Theory of the Effect of Spin-Orbit Coupling on Magnetic Resonance in Some Semiconductors. Phys. Rev. B 96, 266-279 (1954).

27. Yafet, Y. in Solid State Physics (eds Seitz, F. \& Turnbull, D.) 1-98 (Academic Press, 1963).

28. Masmanidis, C. A., Jaffe, H. H. \& Ellis, R. L. Spin-Orbit Coupling in Organic Molecules. J. Phys. Chem. 79, 2052-2061. Is SN: 0022-3654 (1975). 
29. Yu, Z. G. Spin-Orbit Coupling, Spin Relaxation, and Spin Diffusion in Organic Solids. Phys. Rev. Lett. 106, 106602. IS S: 0031-9007, 1079-7114 (2011).

30. Yu, Z. G. Spin-Orbit Coupling and Its Effects in Organic Solids. Phys. Rev. B 85, 115201 (2012).

31. Van Lenthe, E., Snijders, J. G. \& Baerends, E. J. The Zero-order Regular Approximation for Relativistic Effects: The Effect of Spin-Orbit Coupling in Closed Shell Molecules. J. Chem. Phys. 105, 6505-6516. IS N : 0021-9606 (1996).

32. Pantazis, D. A., Chen, X.-Y., Landis, C. R. \& Neese, F. All-Electron Scalar Relativistic Basis Sets for Third-Row Transition Metal Atoms. J. Chem. Theory Comput. 4, 908-919 (2008).

33. Adamo, C. \& Barone, V. Toward Chemical Accuracy in the Computation of NMR Shieldings: The PBE0 Model. Chem. Phys. Lett. 298, 113-119 (1998).

34. Valiev, M. et al. NWChem: A Comprehensive and Scalable Open-Source Solution for Large Scale Molecular Simulations. Comput. Phys. Commun. 181, 1477-1489 (2010).

35. Yu, Z. G. Microscopic Theory of Electron Spin Relaxation in N@ C60. Phyical Rev. B 77, 821-6 (2008).

36. Nuccio, L. et al. Importance of Spin-Orbit Interaction for the Electron Spin Relaxation in Organic Semiconductors. Phys. Rev. Lett. 110, 216602 (2013).

37. Harmon, N. J. \& Flatté, M. E. Distinguishing Spin Relaxation Mechanisms in Organic Semiconductors. Phys. Rev. Lett. 110, 176602 (2013).

38. Atherton, N. M. Principles of Electron Spin Resonance (Ellis Horwood Limited, 1993).

39. Kirby, N. M. et al. A Low-Background-Intensity Focusing Small-Angle X-Ray Scattering Undulator Beamline. J. Appl. Crystallogr. 46, 1670-1680 (2013).

40. Ilavsky, J. Nika: Software for Two-Dimensional Data Reduction. J. Appl. Crystallogr. 45, 324-328 (2012).

41. Rivnay, J. et al. Unconventional Face-on Texture and Exceptional in-Plane Order of a High Mobility n-Type Polymer. Adv. Mater. 22, 4359-4363 (2010).

42. Rivnay, J. et al. Drastic Control of Texture in a High Performance N-Type Polymeric Semiconductor and Implications for Charge Transport. Macromolecules 44, 5246-5255 (2011).

43. Giussani, E., Fazzi, D., Brambilla, L., Caironi, M. \& Castiglioni, C. Molecular Level Investigation of the Film Structure of a High Electron Mobility Copolymer via Vibrational Spectroscopy. Macromolecules 46, 2658-2670 (2013).

44. Lemaur, V. et al. On the Supramolecular Packing of High Electron Mobility Naphthalene Diimide Copolymers: The Perfect Registry of Asymmetric Branched Alkyl Side Chains. Macromolecules 46, 8171-8178 (2013).

45. Mayo, S. L., Olafson, B. D. \& Goddard, W. A. DREIDING: A Generic Force Field for Molecular Simulations. J. Phys. Chem. 94, 8897-8909. Is s N: 0022-3654 (1990).

46. Olivier, Y. et al. 25th Anniversary Article: High-Mobility Hole and Electron Transport Conjugated Polymers: How Structure Defines Function. Adv. Mater. 26, 2119-2136 (2014).

47. Besler, B. H., Merz, K. M. \& Kollman, P. A. Atomic Charges Derived from Semiempirical Methods. J. Comput. Chem. 11, 431-439. IS S N: 1096-987X (1990). 
48. Singh, U. C. \& Kollman, P. A. An Approach to Computing Electrostatic Charges for Molecules. J. Comput. Chem. 5, 129-145. Is s N: 0192-8651, 1096-987X (1984).

49. McDonald, N. A. \& Jorgensen, W. L. Development of an All-Atom Force Field for Heterocycles. Properties of Liquid Pyrrole, Furan, Diazoles, and Oxazoles. J. Phys. Chem. B 102, 8049-8059. IS S N: 1520-6106 (1998).

50. Jorgensen, W. L. \& McDonald, N. A. Development of an All-Atom Force Field for Heterocycles. Properties of Liquid Pyridine and Diazenes. Journal of Molecular Structure: THEOCHEM. A Faithful Couple: Qualitative and Quantitative Understanding of Chemistry 424, 145-155. IS S N: 0166-1280 (1998).

51. Jorgensen, W. L., Maxwell, D. S. \& Tirado-Rives, J. Development and Testing of the OPLS All-Atom Force Field on Conformational Energetics and Properties of Organic Liquids. J. Am. Chem. Soc. 118, 11225-11236. IS N N 0002-7863 (1996).

52. Jorgensen, W. L. \& Tirado-Rives, J. The OPLS [Optimized Potentials for Liquid Simulations] Potential Functions for Proteins, Energy Minimizations for Crystals of Cyclic Peptides and Crambin. J. Am. Chem. Soc. 110, 1657-1666. Is s n: 0002-7863 (1988).

53. Bussi, G., Donadio, D. \& Parrinello, M. Canonical Sampling through Velocity Rescaling. The Journal of Chemical Physics 126, 014101. Is S N: 0021-9606 (2007).

54. Berendsen, H. J. C., Postma, J. P. M., van Gunsteren, W. F., DiNola, A. \& Haak, J. R. Molecular Dynamics with Coupling to an External Bath. The Journal of Chemical Physics 81, 3684-3690. IS S N: 0021-9606 (1984).

55. Stewart, J. J. P. Optimization of Parameters for Semiempirical Methods I. Method. J. Comput. Chem. 10, 209-220. ISSN: 1096-987X (1989).

56. DeLongchamp, D. M., Kline, R. J., Fischer, D. A., Richter, L. J. \& Toney, M. F. Molecular Characterization of Organic Electronic Films. Adv. Mater. 23, 319-337 (2011).

57. Gao, J., Thomas, A. K., Johnson, R., Guo, H. \& Grey, J. K. Spatially Resolving Ordered and Disordered Conformers and Photocurrent Generation in Intercalated Conjugated Polymer/Fullerene Blend Solar Cells. Chem. Mater. 26, 4395-4404. Is s n: 0897-4756 (2014).

58. Khan, A. I. et al. Equilibrium Molecular Dynamics (MD) Simulation Study of Thermal Conductivity of Graphene Nanoribbon: A Comparative Study on MD Potentials. Electronics 4, 1109-1124 (2015).

59. Pronk, S. et al. GROMACS 4.5: A High-Throughput and Highly Parallel Open Source Molecular Simulation Toolkit. Bioinformatics 29, 845-854. IS S : 1367-4811 (2013).

60. Humphrey, W., Dalke, A. \& Schulten, K. VMD: Visual Molecular Dynamics. Journal of Molecular Graphics 14, 33-38. IS S N: 0263-7855 (1996). 